Advances in Magneto-Optics, Proc. Int. Symp. Magneto-Optics, J. Magn. Soc. Jpn. Vol. 11, Supplement, No. S1 (1987) 385-388. (C) 1987 by The Magnetics Society of Japan

\title{
AN APPROACH TOWARDS A SINGLE MODE GARNET RIB WAVEGUIDE CALCULATIONS AND EXPERIMENTS
}

\section{P. FRIEZ, J. MACHUI, and P-L MEUNIER}

Lab. Magnéto-optique, Laboratoire Central de Recherches, THOMSON-CSF Domaine de Corbeville, 91401 ORSA Y, France

\section{ABSTRACT}

A monomode optical isolator depends upon the achievement of a substituted YIG waveguide.

Computer simulations were used to study the propagation behaviour of polarised monochromatic light beams in guiding garnet structures. We used two approches : coupling analysis for TE and TM modes propagating in the upper layer of a planar YIG1/YIG2/GGG structure and a finite element analysis for two-dimensional structures, e.g. partially etched trapezoidal rib waveguides.

We achieved single and double layer rib waveguides by ion beam milling and investigated the influence of the incident angle of the ion beam and the milling-rate in order to master the roughness of the edges.

\section{INTRODUCTION}

Isolators can be used in optical communication systems to decouple the laser source from the transmission medium. The light reflected in the fibre network might else perturbe the laser's performance.

We are interested in a second generation of integrated isolators that makes use of Faraday's effect in ferrimagnetic materials, $i . e$. of the interaction between a magnetic field $M$, proper to the material, and the guided light.

The guiding structures we studied consist of substituted Yttrium Iron Garnet (YIG) substrate. We furthermore restricted ourselves to one - standard - wavelength $\lambda_{0}=1,55 \mu \mathrm{m}$.

This combination is rather favourable as one has relatively little propagation losses and a strong Faraday effect of up to $200^{\circ} / \mathrm{cm}$. Finally YIG and GGG crystals show strong structural similarities and we can grow satisfactory YIG layers using liquid phase epitaxy.

The above choice being made, we are left with basically two free parameters : we can vary the substitution in our YIG (Gd,45. $\mathrm{Y}_{2.55} \quad \mathrm{Ga} \mathrm{Fe}_{5-y} \mathrm{O}_{12}$ with $0.8 \leq \mathrm{y} \leqslant 1.0$, which allows e.g. for a modification of its refraction index $\mathrm{n}_{Y \mid G} \mathrm{y}$ of up to about $\left.+/-10^{-2}\right)$, and we can play with the geometric structure of our guide. These parameters have to be picked carefully in order to make the device monomodal, to end up with a size that guaranties good coupling with current optical fibres and, finally, to achieve good conversion properties.

The guides we think of are sketched in fig. 4. They allow for the propagation of modes with different alignment of the electro-magnetic field. One talks about $T E$ or $\mathrm{HE}$ modes if the electric field $E$ is essentially parallel to the substrate surface, $i . e . E_{x}>E_{y}$. Inversely, for $H_{x}>H_{y}$ we call them TM or EH modes.

In analogy to the turning of the axis of polarization in bulk isolators, one uses the Faraday effect to convert TE modes into TM modes and vice versa. The magnetic field $M$ in the YIG parallel to the axis of light propagation gives rise to a non reciprocal interaction between TE and TM modes, non reciprocal meaning that the effect does not depend on the orientation of the light bean, but only on the orientation of the magnetic field.

A main obstacle comes from different effective wave numbers $B_{T E}$ and $B_{T M}$ for corresponding TE and TM modes. The resulting phase shift between the modes prohibits efficient conversion, the maximum possible depends on $\Delta B=B_{T E}-B_{T M}$. Using coupling of modes theorie with a coupling constant $K_{F}$ from Faraday's effect, we find for the maximum of mode conversion in terms of intensity

$$
R_{\max }=4 K_{F}^{2} /\left(4 K_{F}^{2}+\Delta B^{2}\right)
$$

Clearly for $100 \%$ conversion we want $\Delta B$ to vanish.

Introducing effective refraction indices $n_{T E}, n_{T M}$ for $T E$ and $T M$ modes with effective wave numbers $B_{T E}, B_{T M}$ respectively by

$$
n_{\mathrm{TE}}=B_{\mathrm{TE}} \lambda_{0} / 2 \pi, \quad n_{\mathrm{TM}}=B_{\mathrm{TM}} \lambda_{0} / 2 \pi
$$

we can rewrite $\Delta B$ using $\Delta \mathrm{n}=\mathrm{n}_{\mathrm{TE}}-\mathrm{n}_{\mathrm{TM}}$ as

$$
\Delta B=\Delta \mathrm{n} 2 \pi / \lambda_{0}
$$

Looking more closely at $\Delta \mathrm{n}$ one finds that it combines a number of effects. There is, to begin with, a $\Delta n$ from the epitaxial growth process of the YIG layer, but $\Delta \mathrm{n}$ vanishes when we reheat the sample." This leaves us with $\Delta \mathrm{n}$ from a difference of lattice parameters between the GGG substrate and the YIG layer, and eventually $\Delta \mathrm{n}_{\mathrm{g}}$ from the geometry we chose for the guide. As we want

$$
\Delta \mathrm{n}=\Delta \mathrm{n}_{\mathrm{g}}+\Delta \mathrm{n}_{1}=0
$$


for good conversion, we should like $\Delta \mathrm{n}_{\mathrm{g}}$ and $\Delta \mathrm{n}_{1}$ to be of the same magnitude but of opposite
signs.

Our structures lead to $\Delta \mathrm{n}_{\mathrm{g}}$ positive, we therefore want $\Delta \mathrm{n}_{1}<0$. The magnitude of $\Delta \mathrm{n}_{1}$ is restricted by the need for good epitaxial growth, but we can achieve $\Delta n_{1}=-.510^{-4}$.

\section{ANALYSIS OF PLANAR STRUCTURES}

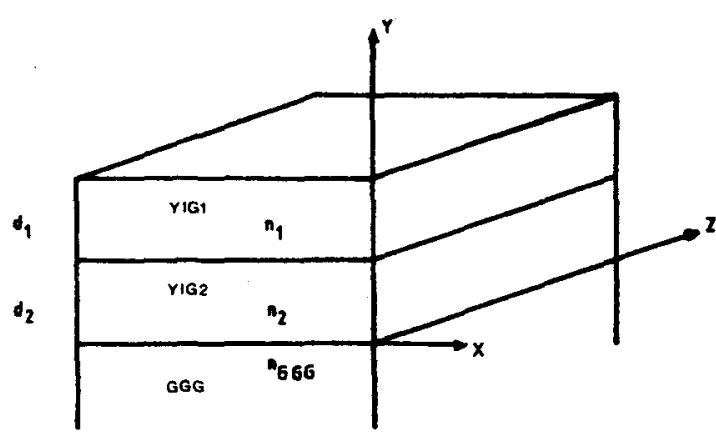

FIG. 1 : Schematic presentation of a double YIG layer on GGG substrate.

We find valuable first informations about our guide from an analysis of planar structures as indicated in fig. 1 for a double layer of YIG on GGG. It is a well established result [1] that single layer guides would have to be very thin $(1.2 \mu \mathrm{m}$ for $n_{Y I G}=2.158$ and $n_{G G G}=1.945$ ) to be monomodal (in the sense that only one TE and one TM mode are guided). This means very bad coupling coefficients with current optical fibres, who demand an aperture of 4 to $6 \mu \mathrm{m}$.

There is a second major drawback of isotropical single layers. They produce a large $\Delta n_{g}$ for the kind of layers we need $\left(\mathrm{e} . \mathrm{g} . \Delta \mathrm{n}_{\mathrm{g}}=.510^{-3}\right.$ for $4 \mu \mathrm{m}$ YIG layers, this means $\mathrm{R}_{M}=3 \%$. for $\mathrm{K}_{\mathrm{F}}=100 \% \mathrm{~cm}$ which is evidently not sufficient).

We therefore looked at multilayer structures and in particular at double layers as indicated in fig. 1 with $n_{1}>n_{2}>n_{G G G}$, i.e. the wave is guided in the upper YIG layer.

We used calculations based on Helmholtz' equation, as derived from Maxwell's equations. In our special case we have $\partial / \partial x=0$ and we fixed $O z$ as the propagation direction. Furthermore the air and the GGG substrate is considered infinit for $y=+/-\infty$, and all materials are isotropic.

We used separation of variables for $\Phi_{1}(y)=E_{x}(y)$ and $\Phi_{2}(y)=H_{x}(y)$ for TE and TM modes respectively. The result combined with continuity for $\Phi_{1}, \Phi_{1}^{\prime}$ and $\Phi_{2}^{\prime} / n^{2}$ at material interfaces leads to the following equations [2] for the effective refraction index $n_{\text {eff }}=B_{\text {eff }} \lambda_{0} / 2 \pi$ of $T E$ and $T M$ modes

- for modes guided in the upper layer $\left(n_{2} \leq n_{\text {eff }}<n_{1}\right)$

$\operatorname{tg}\left(\bar{B}_{1} d_{1}-\phi_{01}-m_{1} \pi\right)-\eta_{21} \operatorname{cotanh}\left(\bar{B}_{2} d_{2}+\psi_{23}\right)=0$

with $\bar{B}_{2}=\sqrt{B_{e f f}^{2}-n_{2}^{2} k_{0}^{2}}, k_{0}=2 \pi / \lambda_{0}$,

- for modes guided by both layers $\left(n_{G G G} \leq n_{\text {eff }}<n_{2}\right.$ )

$\operatorname{tg}\left(\bar{B}_{1} d_{1}-\phi_{o 1}-m_{1} \pi\right)+\eta_{21} \operatorname{tg}\left(\bar{B}_{2} d_{2}-\phi_{32}-m_{2} \pi\right)=0$

with $\bar{B}_{2}=\sqrt{n_{2}^{2} k_{0}^{2}-B_{e f f}^{2}}$

where $\bar{B}_{1}=\sqrt{n_{1}^{2} k_{0}^{2}-B_{\text {eff }}^{2}}, \quad \bar{B}_{0}=\sqrt{B_{\text {eff }}^{2}-k_{o}^{2}}, \quad \bar{B}_{3}=\sqrt{B_{e f f}^{2}-n_{G G G}^{2} k_{o}^{2}}$

and $\phi_{01}=\operatorname{tg}^{-1} \eta_{01}, \psi_{23}=\tanh ^{-1} \eta_{23}, \phi_{32}=\operatorname{tg}^{-1} \eta_{32}$

using $\eta_{i j}= \begin{cases}\bar{B}_{i} / \bar{B}_{j} & \text { for } T E \text { modes } \\ n_{j}^{2} \bar{B}_{i} / n_{i}^{2} \bar{\beta}_{j} & \text { for TM modes }\end{cases}$

The number of the mode $m$ is given by $m_{1}$ in (5) and the following rule for the case of (6) :

Given $T_{1}=\left|\bar{B}_{1} d_{1}-\phi_{o 1}-m_{1} \pi\right|$ and $T_{2}\left|\bar{B}_{2} d_{2}-\phi_{32}-m_{2}\right|$

$-\mathrm{m}=\mathrm{m}_{1}+\mathrm{m}_{2}$, if $\mathrm{T}_{1} \leq \pi / 2$ and $\mathrm{T}_{2} \leq \pi / 2$,

$-m=m_{1}+m_{2}+1$, if one of $T_{1}$ or $T_{2}$ is greater than $\pi / 2$,

$-\mathrm{m}=\mathrm{m}_{1}+\mathrm{m}_{2}+2$, if $\mathrm{T}_{1}>\pi / 2$ and $\mathrm{T}_{2}>\pi / 2$.

Fig. 2 shows the effective refraction index of the first two TE and TM modes for varying thickness $d_{1}$ of the guiding YIG layer, $d_{2}$ being fixed at $4 \mu \mathrm{m}$.

The critical thickness for the upper YIG layer to be monomodal is $d_{\text {c }}=6.5 \mu \mathrm{m}$ for $\mathrm{n}=2.158$ and $n_{2}=2.15$. We find furthermore $\Delta n_{g}=.610^{-4}$ for $d_{1}=d$ and $\Delta n_{g}=1.710^{-4}$ for $d_{1}=4 \mu m$. These ${ }^{2}$ values are of an order of magnitude comparable with $\Delta \mathrm{n}_{1}$ and we can hope to end up with a satisfactory total value for $\Delta \mathrm{n}$.

The field destribution of the first $\mathrm{TE}$ mode along the $y$ axis is shown in fig. 3 for $d_{1}=d_{2}=$ $4 \mu \mathrm{m}$. We may expect reasonable coupling with optical fibres of a diameter of $6 \mu \mathrm{m}$. 


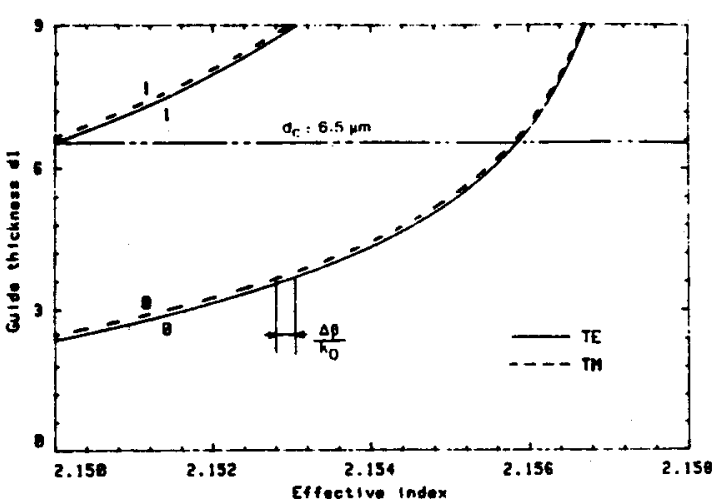

FIG. 2 : Effective index of the first two TE and TM modes for variable thickness of the upper layer $d_{1}$ and $d_{2}=4 \mu \mathrm{m}$ with $n_{1}=2.158$ and $n_{2}=2.15$. Only one mode propagates in the upper layer for $d_{1}<d_{c}$.

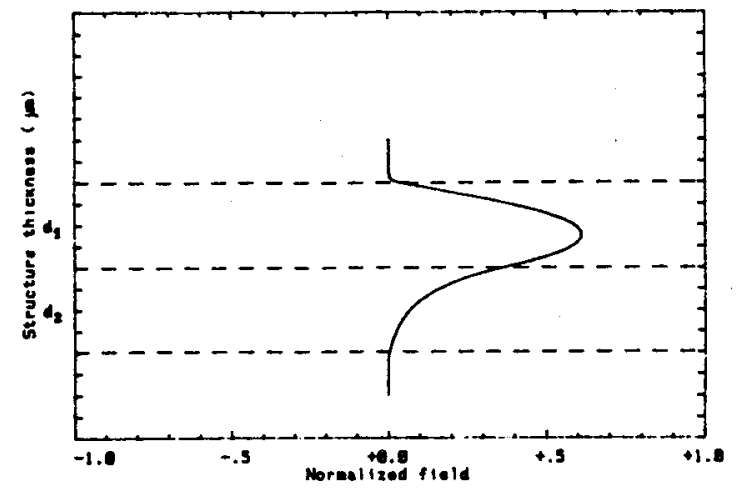

FIG. 3 : Field distribution of the first TE mode in the double layer with $\mathrm{d}_{1}=\mathrm{d}_{2}=4 \mu \mathrm{m}$.

\section{FINITE ELEMENT ANALYSIS}

A purely one-dimensional analysis, though essential for an improved understanding of principal difficulties, cannot be sufficient. Our guide needs a two-dimensional structure to avoid excessive lateral losses, as well as one would like to use these losses to disperse unwanted secondary modes.

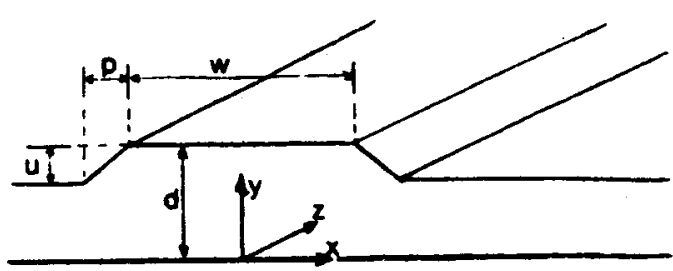

FIG. 4 : Sketch of a partially etched rib waveguide

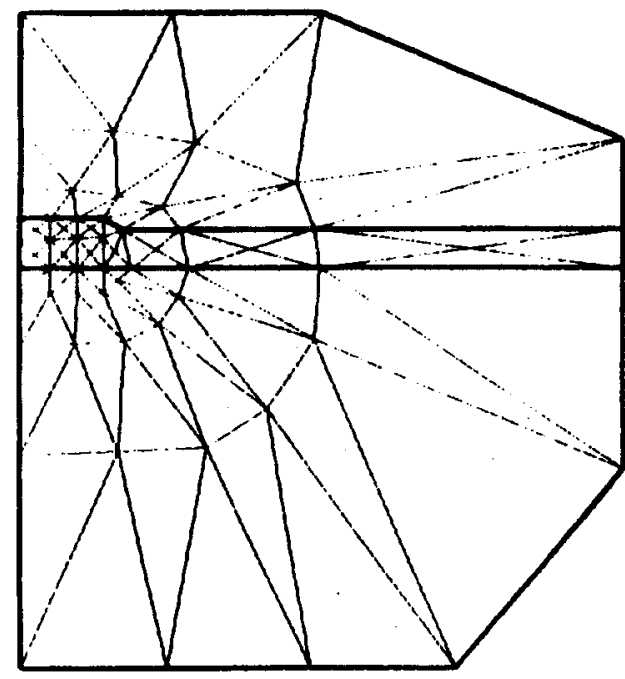

FIG. 5 : Mesh for a single layer rib waveguide. We used second order elements.

\begin{tabular}{cccc}
\hline & 4.0 & 4.0 & \\
$w$ & 4.5 & 4.5 & 9.0 \\
0 & .7 & 1.5 & 9.0 \\
\hline$n_{T E}$ & .5 & 1.07 & 1.0 \\
$n_{\text {TE }}$ & 2.142 & 2.148 & 2.144 \\
$n_{\text {TE } 3}$ & 2.137 & 2.1412 & 2.142 \\
\hline$n_{c}$ & 2.134 & 2.137 & 2.138 \\
\hline & 2.148 & 2.1415 & 2.148
\end{tabular}

TAB. 1 : Comparison of effective indices for the first three $\mathrm{TE}$ modes of different guide geometries with $n_{Y I G}=2.158, n_{G G G}=1.945$.

One can nevertheless take for granted that again double layers show a better behaviour and we think to use the above means in order to optimize the structure we shall employ.
The finite element approach we used stems from Mabaya et al. [3], who showed its validity in particular for rectangular guides. It exploits $E_{X} \gg E_{Y}$ for $\mathrm{TE}$ (or $\mathrm{HE}$ ) modes and $\mathrm{H}_{\mathrm{x}} \gg \mathrm{H}_{\mathrm{y}}$ for $\mathrm{TM}$ (or $\mathrm{EH}$ ) modes setting

$$
\mathrm{E}_{\mathbf{y}}=0 \text { and } \mathrm{H}_{\mathrm{y}}=0
$$

respectively. We shall thus continue to talk about TE and TM modes, also to avoid confusion.

Fig. 4 shows a sketch of the rib waveguide we analysed. The guide is partially etched and a single YIG layer is represented. We exploited the symmetry of the problem, and fig. 5 shows the mesh used for a single layer guide with $d=4 \mu \mathrm{m}, u=.7 \mu \mathrm{m}$ and $\mathrm{p}=.5 \mu \mathrm{m}$. Note that the $x$-axis is amplified by a factor of 2 .

Fig. 6 shows the first $3 \mathrm{TE}$ modes we found using $\mathrm{n}_{Y \mid G}=2.158$ and $\mathrm{n}_{\mathrm{GGG}}=1.945$. The first mode seems to be guided by our partially etched guide, the second antisymmetrical one already seems to disperse to a considerable extent sideways and the third calculated one finally indicates that one may expect no higher order modes to be guided.

We can now go back to one-dimensional analysis. The effective index $n_{c}$ of the first TE mode guided in a YIG layer of thickness $\mathrm{d}-\mathrm{u}$ defines some cut-off condition for our guide. Tab. 1 compares the effective refraction indices of the first three $\mathrm{TE}$ modes of. three different guide geometries to $\mathrm{n}_{\mathrm{c}}$. The relation between $n_{c}$ and the respective $n_{e f f}$ gives an idea of the sideward leakyness of every mode, and helps to find a good compromise between low losses and monomodal behaviour.

The other point of interest concerns $\Delta n_{q}$ for the first $\mathrm{TE}$ and $\mathrm{TM}$ modes of differents structures.

First calculations of multilayer structures show a $\Delta n_{\mathrm{g}}$ of less than $10^{-4}$. These values approach the order of magnitude of numerical errors stemming from the approximation (10) we made, from the algorithms we employed or from the meshes we used (we compared different meshes and found an error of some $10^{-5}$ for the first modes). 


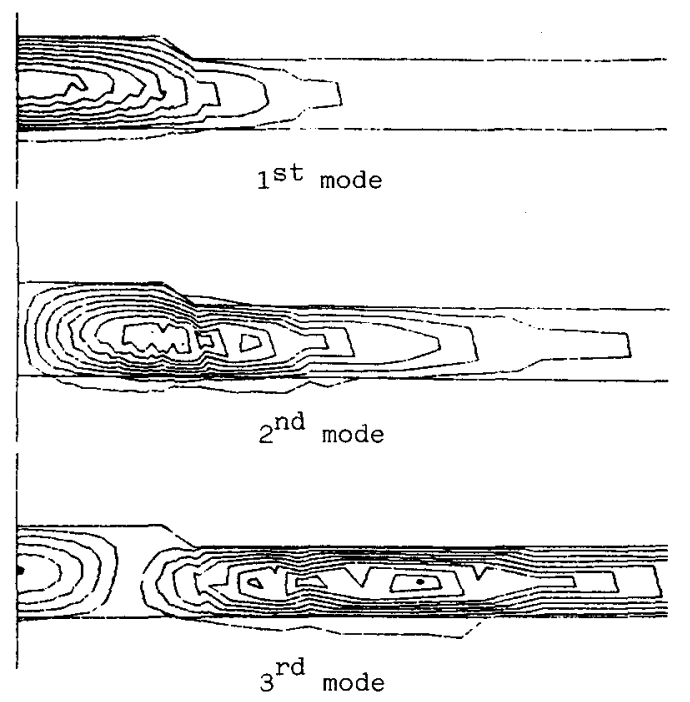

FIG. $6: \mathrm{E}_{\mathrm{X}}$ component of the first three $\mathrm{TE}$ mode in a single layer guide

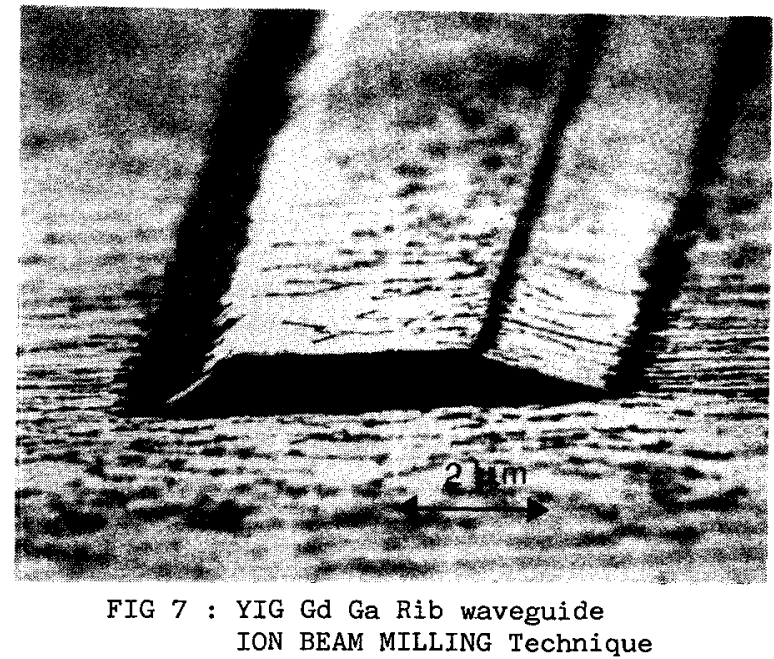

\section{TECHNOLOGY AND MEASUREMENTS}

We employed an ion milling process to produce the rib waveguides whose shape and dimensions are estimated by the former calculations. We wanted to avoid wet techniques and find a technology compatible with future laser processing in view of a total integration on one chip [4].

Having tried a number of masking techniques we ended up with a regular photoresist layer of $2 \mu \mathrm{m}$ for 1350 SHIPLEY and $1 \mu \mathrm{m}$ for 1370 SHIPLEY. The mask was heated at $100^{\circ} \mathrm{C}$ to $150^{\circ} \mathrm{C}$ in order to harden it for future milling. Studies were carried out to straighten the upper edges of the overlay, key pattern to obtain steep edges of the guides. Needing differently substituted YIG layers, we investigated the ion beam milling rate of YIG according to the Ga $\mathrm{rate}(\mathrm{Gd}, 45 \mathrm{Ga} Y \mathrm{YIG}$ with $.6 \leq x \leq 1.1)$ and to the incident angle $\alpha$ of the beam $\left(\alpha=0^{\circ}\right.$ or $\left.35^{\circ}\right)$. The etching rate is almost similar for the different substitutions depending upon the ion density and the incident beam $\alpha$. The ratio: milling rate AZ 1350/milling rate YIG is going from 1 to 4 . These results allow us to tune the differential etching rate to achieve the desired rib waveguide. Further studies are being performed on the ripple of the guides and will be published later.

We achieved up to 5000 to $9000 \AA$ high and $7 \mu \mathrm{m}$ wide ribs. The ripple of the edges was compatible with the wavelength $(1.3 \mu \mathrm{m}$ to $1.5 \mu \mathrm{m})$, the shape of the cross section fits a trapezoid rather than a rectangle, as was taken into account in the above calculations.

To characterize the guide, both ends had to be cut and polished. Standard means of integrated optics technology were used to achieve a steep cross section without destroying the rib.

One of the measured guides gives the following results : losses $\simeq 5 \mathrm{~dB} / \mathrm{cm}$. It's a simple layer guide $4.9 \mu \mathrm{m}$ thick, the $\mathrm{rib}$ is $7200 \AA \mathrm{high}$ and $11 \mu \mathrm{m}$ wide.

We finally produced a double layer rib waveguide with $\mathrm{d}_{1}=3,7 \mu \mathrm{m}, \mathrm{d}_{2}=2,9 \mu \mathrm{m}\left(\mathrm{Gd}_{0,45} \mathrm{Ga} 0.8\right.$ $\left.\mathrm{YIG} / \mathrm{Gd}_{0,45} \mathrm{Ga}_{0,9} \mathrm{YIG}\right), 9 \mu \mathrm{m}$ wide and $9000 \AA \mathrm{high}\left(\mathrm{n}_{1}=2,1565\right.$ and $\left.\mathrm{n}_{2}=2,1511\right)$. This guide shows a monomodal behaviour and its losses remain inferior to $5 \mathrm{~dB} / \mathrm{cm}$.

\section{CONCLUSION}

The technological process sketched above and an improved understanding of physical mechanisms allow a deeper insight into the problems YIG waveguides offer. The first encouraging results obtained let us nevertheless hope to achieve a monomode isolator of a good conversion rate and reasonable dimensions using YIG layers on GGG substrates.

\section{ACKNOWLEDGEMENTS}

We should like to thank Mrs Magna, Ms Papillon and Mr Soufaché for their valuable technical advices and assistance, as well as Mr Bourbin for helpful physical discussions.

This work was sponsored by the Direction des Affaires Industrielles Internationales.

\section{REFERENCES}

1. J.P Castera : Doctorat de 3ème cycle, Université Paris XI (1977).

2. M.J. Sun and M.W. Muler : Appl. Opt. 16, pp 814-815 (1977).

3. N. Mabaya, P.E. Lagasse and P. Vandelbulke : IEEE Trans., MTT-29, pp 600-605 (1981).

4. M. Razeghi, P-L Meunier, P. Maurel, JAP. V 59 - No.6, pp 2261-2263. 